\title{
Relationship between extravascular lung water and severity categories of acute respiratory distress syndrome by the Berlin definition
}

Shigeki Kushimoto ${ }^{1 *}$, Tomoyuki Endo ${ }^{2}$, Satoshi Yamanouchi', Teruo Sakamoto ${ }^{3}$, Hiroyasu Ishikura ${ }^{4}$, Yasuhide Kitazawa ${ }^{5}$, Yasuhiko Taira ${ }^{6}$, Kazuo Okuchi $^{7}$, Takashi Tagami ${ }^{8}$, Akihiro Watanabe ${ }^{8}$, Junko Yamaguchi ${ }^{9}$, Kazuhide Yoshikawa ${ }^{10}$, Manabu Sugita ${ }^{11}$, Yoichi Kase ${ }^{12}$, Takashi Kanemura ${ }^{13}$, Hiroyuki Takahashi ${ }^{14}$, Yuuichi Kuroki ${ }^{15}$, Hiroo Izumino ${ }^{16}$, Hiroshi Rinka ${ }^{17}$, Ryutarou Seo ${ }^{18}$, Makoto Takatori ${ }^{19}$, Tadashi Kaneko ${ }^{20}$, Toshiaki Nakamura ${ }^{21}$, Takayuki Irahara ${ }^{22}$ and Nobuyuki Saito ${ }^{23}$, for the PiCCO Pulmonary Edema Study Group

\begin{abstract}
Introduction: The Berlin definition divides acute respiratory distress syndrome (ARDS) into three severity categories. The relationship between these categories and pulmonary microvascular permeability as well as extravascular lung water content, which is the hallmark of lung pathophysiology, remains to be elucidated. The aim of this study was to evaluate the relationship between extravascular lung water, pulmonary vascular permeability, and the severity categories as defined by the Berlin definition, and to confirm the associated predictive validity for severity.
\end{abstract}

Methods: The extravascular lung water index (EVLWi) and pulmonary vascular permeability index (PVPI) were measured using a transpulmonary thermodilution method for three consecutive days in 195 patients with an EVLWi of $\geq 10 \mathrm{~mL} / \mathrm{kg}$ and who fulfilled the Berlin definition of ARDS. Collectively, these patients were seen at 23 ICUs. Using the Berlin definition, patients were classified into three categories: mild, moderate, and severe.

Results: Compared to patients with mild ARDS, patients with moderate and severe ARDS had higher acute physiology and chronic health evaluation II and sequential organ failure assessment scores on the day of enrollment. Patients with severe ARDS had higher EVLWi (mild, 16.1; moderate, 17.2; severe, 19.1; $P<0.05)$ and PVPI $(2.7 ; 3.0 ; 3.2 ; P<0.05)$. When categories were defined by the minimum $\mathrm{PaO}_{2} / \mathrm{FlO}_{2}$ ratio observed during the study period, the 28-day mortality rate increased with severity categories: moderate, odds ratio: 3.125 relative to mild; and severe, odds ratio: 4.167 relative to mild. On independent evaluation of 495 measurements from 195 patients over three days, negative and moderate correlations were observed between EVLWi and the $\mathrm{PaO}_{2} / \mathrm{FIO}_{2}$ ratio $(r=-0.355, P<0.001)$ as well as between $\mathrm{PVPI}$ and the $\mathrm{PaO}_{2} / \mathrm{FIO}_{2}$ ratio $(r=-0.345, P<0.001)$. ARDS severity was associated with an increase in EVLWi with the categories (mild, 14.7; moderate, 16.2; severe, 20.0; $P<0.001)$ in all data sets. The value of PVPI followed the same pattern $(2.6 ; 2.7$; 3.5; $P<0.001)$.

Conclusions: Severity categories of ARDS described by the Berlin definition have good predictive validity and may be associated with increased extravascular lung water and pulmonary vascular permeability.

Trial registration: UMIN-CTR ID UMIN000003627

\footnotetext{
* Correspondence: kussie@emergency-medicine.med.tohoku.ac.jp 'Division of Emergency Medicine, Tohoku University Graduate School of Medicine, 2-1, Seiryo-machi, Aoba-ku, Sendai, Miyagi 980-8575, Japan Full list of author information is available at the end of the article
} 


\section{Introduction}

Since the first description of acute respiratory distress syndrome (ARDS) by Ashbaugh et al. in 1967 [1], the definition had been continuously reworked until publication of the American-European Consensus Conference (AECC) definition in 1994 [2]. The AECC definition has been widely used for epidemiological studies, clinical trials, and critical care practice. It has facilitated advances in the acquisition of clinical and epidemiological data, leading to improvements in the care for patients with ARDS.

Although many clinical trials have been performed since publication of the AECC definition, several issues regarding the definition have emerged. These include a lack of explicit criteria for defining acute manifestations of the disease, sensitivity of the $\mathrm{PaO}_{2} / \mathrm{FIO}_{2}$ ratio $(\mathrm{P} / \mathrm{F}$ ratio) to different ventilator settings, poor reliability of the chest radiograph criterion, and difficulties in distinguishing hydrostatic edema [3-6]. These criteria are also not sensitive predictors of disease severity or patient outcome [7-11].

Recently, theBerlin definition for ARDS has been published, focusing on feasibility, reliability, validity, and objective evaluation of its performance [12]. The definition includes mild, moderate, and severe ARDS based on the degree of hypoxemia. Progression from one category to another is associated with increased mortality.

ARDS is considered to be a type of acute, diffuse, inflammatory lung injury that leads to increased pulmonary vascular permeability, increased lung weight, and the loss of aerated lung tissue. However, no study has investigated the empirical relationship between a given ARDS stage and pulmonary microvascular permeability or extravascular lung water (EVLW) content [12].

Previous studies have reported various methods of quantifying pulmonary edema $[13,14]$. The double-indicator thermodilution technique allows researchers to measure the amount of EVLW. The in vivo and postmortem gravimetric EVLW values obtained using this method were closely correlated in both animal and human studies $[15,16]$. However, this method is excessively cumbersome and technically challenging for routine clinical application. Hence, the single-indicator technique is used in clinical settings; this method is as sensitive as the double-indicator technique $[17,18]$. Previously, we validated the accuracy of EVLW measurements obtained from postmortem lung samples using the single-indicator technique and defined statistically normal EVLW values, as determined through human autopsy [19]. The transpulmonary thermodilution technique provides an estimation of both EVLW and pulmonary blood volume. The ratio of these two parameters is denoted as the pulmonary vascular permeability index (PVPI). This ratio reflects the degree of pulmonary microvascular permeability [20], which is pathognomonic for ARDS.
The aims of this study were to evaluate the relationship between EVLW and pulmonary vascular permeability as assessed using the transpulmonary single thermodilution technique, on one hand, and severity categories of ARDS according to the Berlin definition in patients with acute respiratory failure, on the other. In addition, the study confirms the predictive validity of the Berlin stages with regard to disease severity and mortality.

\section{Materials and methods}

This is a post hoc, subgroup analysis of a previously reported study conducted to clarify the clinical pathophysiological features of ARDS and to establish quantitative diagnostic criteria [21]. This study was approved by the ethics committee of each of the 23 institutions, and written informed consent was provided by each patient's next of kin. The investigation was registered with the University Hospital Medical Information Network (UMIN) Clinical Trials Registry, UMIN-CTR ID UMIN000003627.

\section{Patients}

Between March 2009 and August 2011, 301 patients were enrolled. The inclusion criteria were: age $\geq 15$ years; a need for mechanical ventilation (expected $>48 \mathrm{~h}$ ) for acute respiratory failure with a $\mathrm{P} / \mathrm{F}$ ratio of $\leq 300 \mathrm{~mm} \mathrm{Hg}$; and bilateral infiltration as determined by chest radiography. The exclusion criteria were as follows: over five days from the onset of acute respiratory failure; chronic respiratory insufficiency; a history of pulmonary resection, pulmonary thromboembolism, severe peripheral arterial disease; a cardiac index of $<1.5 \mathrm{~L} \cdot \mathrm{min}^{-1} \cdot \mathrm{m}^{-2}$; lung contusion and burns; and other causes unsuitable for evaluation with the transpulmonary thermodilution technique.

Among the 301 patients, 35 were excluded and 207 were considered to have acute lung injury (ALI)/ARDS (Figure 1).

The diagnosis of pulmonary edema was established on the basis of the following criteria: (1) the presence of bilateral infiltrates on chest radiographs; (2) a P/F ratio of $\leq 300$ $\mathrm{mm} \mathrm{Hg}$; and (3) an increase in the EVLW indexed to the predicted body weight (EVLWi) of $\geq 10 \mathrm{~mL} / \mathrm{kg}$. Although there is no definitive quantitative criterion for the EVLWi indicative of pulmonary edema, we recently reported that the normal EVLWi value is approximately $7.4 \pm 3.3 \mathrm{~mL} / \mathrm{kg}$ in humans [19]. An increase in the EVLWi of $\geq 10 \mathrm{~mL} / \mathrm{kg}$ was used to define pulmonary edema, as previously reported [22,23].

Among 207 patients determined to have ALI/ARDS, 12 patients with positive end-expiratory pressure (PEEP) $<5$ $\mathrm{cmH}_{2} \mathrm{O}$ who did not require airway pressure release ventilation were excluded. Each of the remaining 195 patients had experienced respiratory failure not fully explained by cardiac failure or volume overload and fulfilled the criteria of the Berlin definition. Based on the Berlin definition, 


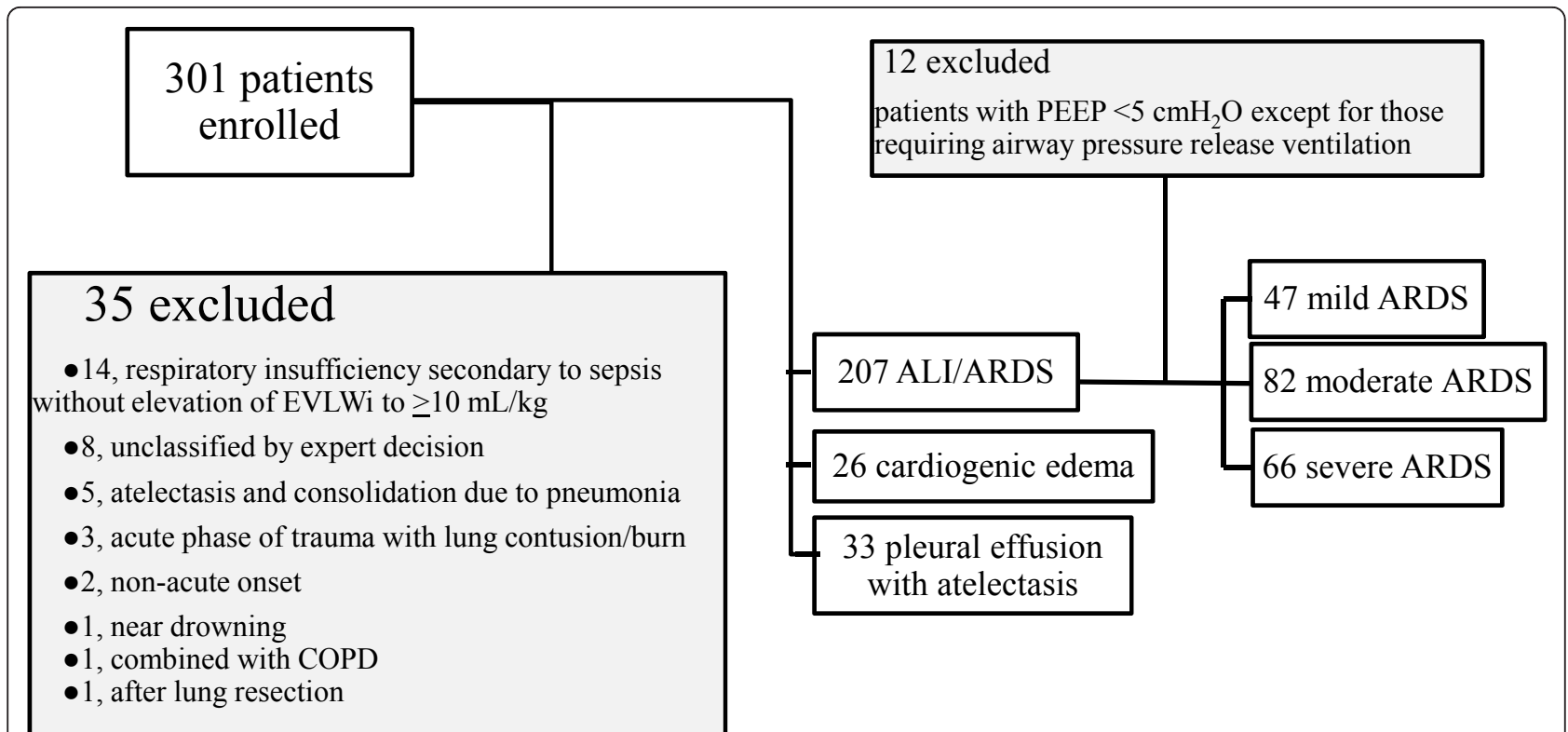

Figure 1 Patient enrollment, exclusion, and classification. ALI/ARDS, acute lung injury/acute respiratory distress syndrome; COPD, chronic obstructive pulmonary disease; EVLWi, extravascular lung water index; PEEP, positive end-expiratory pressure.

patients/respiratory statuses were divided into three categories: mild, moderate, and severe ARDS (Figure 1).

\section{Measurement of EVLWi and pulmonary vascular permeability index}

A thermistor-tipped catheter was connected to the $\mathrm{PiCCO}^{\mathrm{TM}}$ plus or PiCCO ${ }^{\mathrm{TM}} 2$ monitor. This monitor uses a single-thermal indicator technique to calculate the cardiac output, global end-diastolic volume (GEDV), and EVLW. GEDV is calculated as the difference between the intrathoracic thermal volume (ITTV) and pulmonary thermal volume (PTV), which represents the combined enddiastolic volumes of four cardiac chambers. Intrathoracic blood volume (ITBV) was calculated as $1.25 \times$ GEDV 28.4 [17]. EVLW is the difference between the ITTV and ITBV $[17,24]$. PVPI was calculated as the ratio of EVLW to pulmonary blood volume [25-27]. The absolute EVLW value was indexed to predicted body weight [28-33]. The measurements of these parameters have been described in detail previously [21].

\section{Assessment of circulatory/respiratory status, other parameters, and clinical course}

At the time of enrollment (day 0), the patient was evaluated with regard to clinical condition, cause of respiratory insufficiency, acute physiology and chronic health evaluation (APACHE) II score, sequential organ failure assessment (SOFA) score, and lung injury scale (LIS) score $[34,35]$. Echocardiography was performed to measure the left ventricular ejection fraction, left ventricular end- diastolic dimension, interventricular septum thickness, E/A ratio, left atrial dimension, inferior vena cava diameter and associated respiratory variation, the presence of hypo/akinesis, the presence of valvular abnormality, left ventricular systolic/diastolic function, and validity of the thermodilutional hemodynamic assessment. Chest computed tomography (CT) was also conducted on the day of enrollment. B-type natriuretic peptide (BNP) or $N$-terminal pro-BNP was measured on the day of enrollment and daily thereafter.

Circulatory/respiratory status was assessed from the day of enrollment to day 2. The patient's clinical course, including respirator setting, LIS score [36], SOFA score, antithrombin activity level, serum procalcitonin level, daily fluid intake/output and balance, and therapeutic interventions (surgery, antibiotics, steroids, diuretics, renal replacement therapy, and so on) were recorded daily. All patients were followed up for 28 days after enrollment.

\section{Determination of the pathophysiological diagnostic differential for respiratory insufficiency}

At least three experts (specializing in intensive care, respirology, and cardiology) retrospectively determined the pathophysiological mechanism of respiratory insufficiency: (1) ALI/ARDS: increased pulmonary vascular permeability with or without increased pulmonary vascular hydrostatic pressure; (2) cardiogenic edema: increased pulmonary capillary hydrostatic pressure without increased vascular permeability; and (3) pleural effusion 
with atelectasis: no evidence of lung edema secondary to increased hydrostatic pressure or vascular permeability, as previously reported $[25,37]$. For this purpose, the experts carefully scrutinized the patient's medical history; clinical presentation and course; findings on chest CT, radiography, and echocardiography; concentrations of serum BNP or $N$-terminal pro-BNP and procalcitonin; and systemic inflammatory status. They also considered the time course of all the preceding findings, including daily fluid intake/output and the balance and requirement of systemic management and respiratory therapy. This pathophysiological diagnostic procedure was conducted by experts who were completely blinded to the PVPI findings.

\section{Statistical analysis}

Data are presented as medians (interquartile range, IQR). Spearman's rank correlation was used for determining the correlation between variables, and Mann-Whitney's $U$ test was used for assessing the differences between groups. For multiple-group comparisons, the analysis of variance on ranks was used with Tukey's test. The proportions were compared using Pearson's chi-square test. The odds ratios (95\% confidence interval) are reported relative to a reference severity, defined as mild ARDS, for the risk of 28-day mortality. Receiver operating characteristic (ROC) curves were generated for lowest $\mathrm{P} / \mathrm{F}$ ratio, highest PVPI and EVLWi by varying the discriminating threshold of each parameter and the area under the ROC curve for each parameter was calculated. A $P$ value of $<0.05$ was considered significant. All statistical analyses were performed using SPSS 19.0 for Windows (SPSS, Chicago, IL, USA).

\section{Results}

Characteristics of patients with ARDS based on the Berlin definition on the day of enrollment

All 195 patients who presented with respiratory failure not fully explained by cardiac failure or volume overload, who also fulfilled the Berlin definition, were included. The patients were divided into the following three categories on the basis of their respiratory status on the day of enrollment: (1) mild ARDS, (2) moderate ARDS, and (3) severe ARDS. Table 1 shows the patient characteristics as measured on the day of enrollment.

There was no significant difference in age and gender associated with severity of ARDS. On the day of enrollment, both moderate and severe ARDS patients had higher APACHE II and SOFA scores than did patients with mild ARDS, and the positive number of systemic inflammatory response syndrome (SIRS) criteria was also higher in patients with severe ARDS as compared to those with mild or moderate ARDS. Although the levels of PEEP were similar among stages, the level of $\mathrm{FIO}_{2}$ required increased with ARDS severity. EVLWi and PVPI on the day of enrollment were significantly higher in severe ARDS patients. While 28-day mortality was higher in patients with moderate and severe ARDS than in patients with mild ARDS, there was no significant difference in the number of ventilator-free days within 28 days among patients with mild, moderate, or severe ARDS.

\section{ARDS severity as defined by minimal $\mathrm{PaO}_{2} / \mathrm{FIO}_{2}$ ratio and patient outcome}

For this analysis, ARDS severity was determined based on the lowest $\mathrm{P} / \mathrm{F}$ ratio measured during the three-day study period. At 28 days, the level of mortality was higher among

Table 1 Characteristics of patients with ARDS based on the Berlin definition on the day of enrollment.

\begin{tabular}{|c|c|c|c|}
\hline & $\begin{array}{c}\text { mild ARDS } \\
(n=47)\end{array}$ & $\begin{array}{c}\text { moderate ARDS } \\
(n=82)\end{array}$ & $\begin{array}{c}\text { severe ARDS } \\
(n=66)\end{array}$ \\
\hline Age, years & $68(59-78)$ & $68(59-82)$ & $67(53-78)$ \\
\hline Gender (male), \% (n) & $70.2 \%(n=33)$ & $63.4 \%(n=52)$ & $66.7 \%(n=44)$ \\
\hline APACHE II score, points & $19(13-25)$ & $25(20-28)^{*}$ & $25(18-30)^{*}$ \\
\hline SOFA score, points & $9(6-12)$ & $11(9-13)^{*}$ & $11(10-14)^{*}$ \\
\hline SIRS criteria & $2.0(1.0-3.0)$ & $2.0(1.8-3.0)$ & $3.0(2.0-3.0)^{* *}$ \\
\hline $\mathrm{FIO}_{2}$ & $0.5(0.4-0.6)$ & $0.6(0.5-0.8)^{*}$ & $1.0(0.8-1.0)^{*} \#$ \\
\hline PEEP, $\mathrm{cmH}_{2} \mathrm{O}$ & $8(5-10)$ & $10(6-12)$ & $10(6-12)$ \\
\hline $\mathrm{PaO}_{2} / \mathrm{FIO}_{2}$ ratio, $\mathrm{mm} \mathrm{Hg}$ & 247 (217-280) & $148(122-173)^{*}$ & $76(60-90)^{*} \#$ \\
\hline GEDI, mL/m² & $780(662-975)$ & $814(711-925)$ & $730(620-952)$ \\
\hline EVLWi, mL/kg & $16.1(12.2-18.1)$ & $17.2(12.9-22.9)$ & $19.1(14.4-22.9)^{* *}$ \\
\hline PVPI & $2.7(2.2-3.2)$ & $3.0(2.4-3.6)$ & $3.2(2.6-4.1)^{*} \# \#$ \\
\hline $\begin{array}{c}\text { Ventilator-free days } \\
\text { within } 28 \text { days }\end{array}$ & $18(0-20)$ & $9(0-19)$ & $4(0-19)$ \\
\hline 28-day mortality, \% (n) & $23.4 \%(n=11)$ & $43.9 \%(n=36)^{* *}$ & $42.4 \%(n=28)^{* *}$ \\
\hline
\end{tabular}

${ }^{*} P<0.01$ vs. mild ARDS; ${ }^{*} P<0.05$ vs. mild ARDS; \#P<0.01 vs. moderate ARDS; \#\#P<0.05 vs. moderate ARDS. ARDS, acute respiratory distress syndrome; APACHE, acute physiology and chronic health evaluation; SOFA, sequential organ failure assessment; SIRS, systemic inflammatory response syndrome; PEEP, positive end-expiratory pressure; GEDI, global end-diastolic blood volume index; EVLWi, extravascular lung water index; PVPI, pulmonary vascular permeability index. 
patients with moderate or severe ARDS as opposed to those with mild ARDS. Severe ARDS was associated with fewer ventilator-free days than was mild ARDS (Table 2). Table 2 also shows the odds ratio for moderate and severe ARDS relative to mild ARDS: moderate ARDS, odds ratio: $2.824(P=0.048)$; severe ARDS, odds ratio: $4.167(P=$ 0.003).

The area under the curve to predict the outcome for the lowest $\mathrm{P} / \mathrm{F}$ ratio (0.635; confidence interval, 0.556 to 0.715 ) was significantly larger than that for EVLWi $(0.521$; confidence interval, 0.437 to 0.605$)$ and PVPI (0.533; confidence interval, 0.450 to 0.616$)(P<0.05)$.

Relationship among extravascular lung water, pulmonary vascular permeability, and ARDS severity

For this analysis, 495 sets of measurements from 195 patients obtained over the course of three days were independently evaluated and classified as mild, moderate, or severe ARDS according to the Berlin definition.

The results revealed negative and moderate correlations between EVLWi and the $\mathrm{P} / \mathrm{F}$ ratio $(\mathrm{r}=-0.355, P<0.001)$ as well as between PVPI and the P/F ratio ( $r=-0.345$, $P<0.001$ ) in all data sets (Figure 2). ARDS severity was associated with an increase in EVLWi (mild, 14.7 (11.5 to 18.1); moderate, 16.2 (12.6 to 20.9); severe, 20.0 (15.5 to 27.3)) and PVPI (mild, 2.6 (2.0 to 3.1); moderate, 2.7 (2.2 to 3.5 ); severe, 3.5 (2.6 to 4.7$)$ ) (Figure 3 , Table 3 ).

\section{Discussion}

In this study, we demonstrated that the Berlin definition of ARDS effectively predicts mortality, the severity of physiological dysfunction, and organ failure. When using the Berlin definition, ARDS severity is associated with an increase in EVLW and pulmonary vascular permeability as assessed using the transpulmonary single thermodilution method in patients who fulfilled the Berlin definition of ARDS with EVLWi $\geq 10 \mathrm{~mL} / \mathrm{kg}$.

The categories of mild, moderate, and severe ARDS as determined by the Berlin definition are associated with increased mortality and severity. The associated predictive validity for mortality is superior to that of the AECC definition. The relationship between ARDS stage and pulmonary microvascular permeability/EVLW content has not been evaluated, although increased EVLW content secondary to increased pulmonary microvascular permeability is widely considered to be a hallmark of ARDS [12].

The transpulmonary thermodilution single-indicator technique provides an estimation of both EVLW and PVPI [20]. This technique is as sensitive as the doubleindicator technique and frequently used in clinical settings $[17,18]$. Previously, we validated the accuracy of EVLW measurements obtained using the single-indicator technique in postmortem lung samples and defined statistically normal EVLW values in a study of human cadavers [19]. The universal diagnostic criteria for ARDS cannot include the measurement of EVLW and pulmonary vascular permeability, because the procedures for measurement are invasive and not feasible for every institution. In this study, we evaluated the relationship among EVLWi, PVPI and ARDS stages in patients with an EVLWi of $\geq 10 \mathrm{~mL} /$ $\mathrm{kg}$ that was not fully explained by cardiac failure or fluid overload. Negative and moderate correlations were observed between the EVLWi and P/F ratio as well as between the PVPI and P/F ratio. Moreover, EVLWi and PVPI values increased in association with ARDS severity. These results suggest that the ARDS progression outlined by the Berlin definition is associated with increases in EVLW content and pulmonary microvascular permeability, which is the hallmark of lung pathophysiology. It has also been demonstrated that the Berlin definition distinguishes the severity categories of ARDS with good predictive validity for mortality, the severity of physiological derangements, and organ failure.

\section{Limitations}

In this study, a subset of patients meeting the ARDS criteria with respiratory failure of more than five days has

Table 2 Severity of ARDS defined by minimal $\mathrm{PaO}_{2} / \mathrm{FIO}_{2}$ ratio and outcome.

\begin{tabular}{|c|c|c|c|c|}
\hline & \multicolumn{2}{|c|}{$\begin{array}{l}\text { mild ARDS } \\
(n=33)\end{array}$} & $\begin{array}{l}\text { moderate ARDS } \\
\quad(n=83)\end{array}$ & $\begin{array}{c}\text { severe ARDS } \\
(n=79)\end{array}$ \\
\hline \multirow{2}{*}{$\begin{array}{c}\text { Ventilator-free days } \\
\text { within } 28 \text { days } \\
\end{array}$} & \multicolumn{2}{|c|}{$18(1-22)$} & $9(0-19)$ & $0(0-18)^{*}$ \\
\hline & 28-day mortality & $\begin{array}{l}\text { Unadjusted } \\
\text { odds ratio }\end{array}$ & 95\% confidence interval & $P$ value \\
\hline $\begin{array}{l}\text { mild ARDS } \\
(n=33)\end{array}$ & $\begin{array}{l}18.2 \% \\
(n=6)\end{array}$ & 1 & (reference) & \\
\hline $\begin{array}{l}\text { moderate ARDS } \\
\quad(n=83)\end{array}$ & $\begin{array}{l}38.6 \% * * \\
(n=32)\end{array}$ & 2.824 & $1.050-7.590$ & 0.048 \\
\hline $\begin{array}{l}\text { severe ARDS } \\
\quad(n=79)\end{array}$ & $\begin{array}{l}48.1 \% \%^{* *} \\
(n=38)\end{array}$ & 4.167 & $1.553-11.236$ & 0.003 \\
\hline
\end{tabular}

\footnotetext{
${ }^{*} P<0.01$ vs. mild ARDS; ${ }^{* *} P<0.05$ vs. mild ARDS. ARDS, acute respiratory distress syndrome.
} 

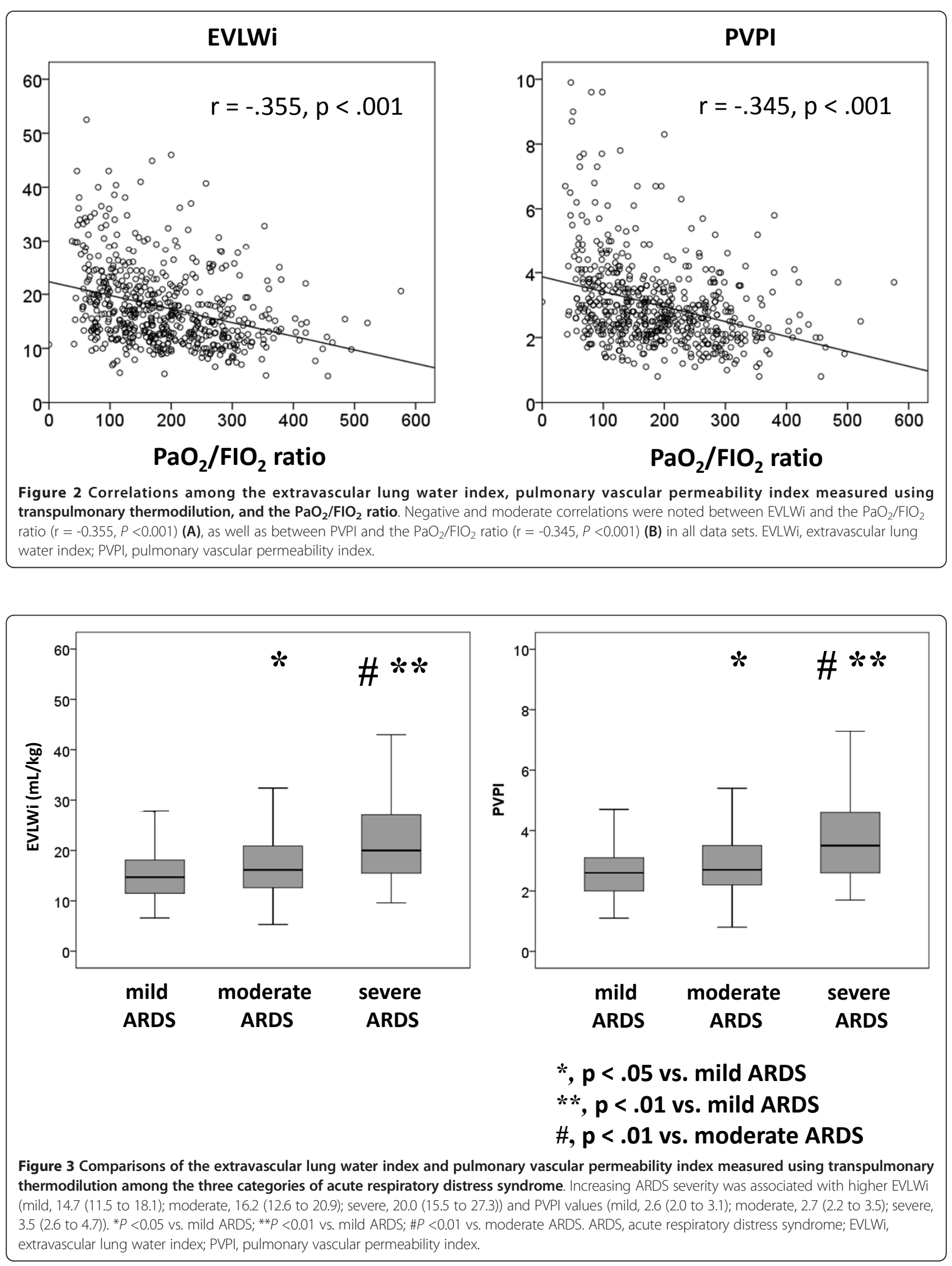
Table 3 EVLWi and PVPI in patients with ARDS based on the Berlin definition during a three-day observation period.

\begin{tabular}{cccc}
\hline & $\begin{array}{c}\text { mild ARDS } \\
(\boldsymbol{n}=\mathbf{1 5 2})\end{array}$ & $\begin{array}{c}\text { moderate ARDS } \\
(\boldsymbol{n}=\mathbf{2 3 0})\end{array}$ & $\begin{array}{c}\text { severe ARDS } \\
(\boldsymbol{n}=\mathbf{1 1 3})\end{array}$ \\
\hline $\mathrm{EVLWi}, \mathrm{mL} / \mathrm{kg}$ & $14.7(11.5-18.1)$ & $16.2(12.6-20.9)^{* *}$ & $20.0(15.5-27.3)^{* \#}$ \\
$\mathrm{PVPI}$ & $2.6(2.0-3.1)$ & $2.7(2.2-3.5)^{* *}$ & $3.5(2.6-4.7)^{*} \#$ \\
\hline
\end{tabular}

${ }^{*} P<0.01$ vs. mild ARDS; ${ }^{* *} P<0.05$ vs. mild ARDS; $\# P<0.01$ vs. moderate ARDS. ARDS, acute respiratory distress syndrome; $E V L W i$, extravascular lung water index; PVPI, pulmonary vascular permeability index.

been excluded. However, the Berlin definition includes patients with respiratory failure within one week of new or worsening respiratory failure. Therefore, a subset of patients meeting the ARDS criteria as diagnosed by the Berlin definition with respiratory failure of more than five days were excluded from the study. The associated results may not fully reflect the characteristics and pulmonary pathophysiology of patients with ARDS as set out by the Berlin definition.

The mechanisms underlying respiratory insufficiency (for example, permeability, pulmonary edema, cardiogenic edema, and/or pleural effusion with atelectasis) were defined by expert consensus; hence, a subjectivity bias cannot be completely ruled out. Nonetheless, only patients who were considered eligible by all the experts were included in the final analysis.

Among 207 patients diagnosed with ARDS in a previous prospective observational study, 12 patients with PEEP $<5 \mathrm{cmH}_{2} \mathrm{O}$ but who did not require airway pressure release ventilation (APRV) were excluded. Patients managed using APRV with PEEP $<5 \mathrm{cmH}_{2} \mathrm{O}$ were included, because their airway pressure was kept higher during respiratory management. This means that some of the patients enrolled in this analysis did not completely fulfill the Berlin definition.

Pulmonary inflammation, that is, pneumonia, may affect the results obtained using the thermodilution technique. Inflamed cells and purulent matter, including microabscesses, might increase lung weight despite increased levels of EVLW. Further evaluation may be required to assess patients with ARDS secondary to direct injury caused by pneumonia.

An EVLWi of $\geq 10 \mathrm{~mL} / \mathrm{kg}$ was used for defining pulmonary edema in this study, as reported previously. Although no definitive quantitative criteria for pulmonary edema were established, this value was selected on the basis of the value found in our recent human autopsy study and those used for defining pulmonary edema in previously reported studies. Lowering the EVLWi value for pulmonary edema could have led to the inclusion of patients with less severe pulmonary edema, which may have influenced the results.
In this study, each patient was managed with a PiCCO system for transpulmonary thermodilution monitoring of his circulatory/respiratory status. Therefore, patients with less severe ARDS may not have been selected for enrollment. Of 35 excluded patients, 14 were judged to have respiratory failure secondary to sepsis-induced increases in pulmonary vascular permeability but had to be excluded because they had previously presented EVLWi $<10 \mathrm{~mL} / \mathrm{kg}$ [21]. Nonetheless, there may exist a considerable number of patients meeting ARDS criteria with 'normal' EVLWi [38]. The inclusion and exclusion criteria used represent potential limitations of the study.

\section{Conclusions}

The severity categories of ARDS laid out by the Berlin definition may be associated with increased EVLW and pulmonary microvascular permeability in patients who fulfilled the Berlin definition of ARDS with EVLWi $\geq 10$ $\mathrm{mL} / \mathrm{kg}$, which is the hallmark of lung pathophysiology. Moreover, this staging has good predictive validity for mortality, the severity of physiological derangements, and organ failure. These relationships require confirmation through further clinical research and critical care practice.

\section{Key messages}

- The categories of acute respiratory distress syndrome laid out by the Berlin definition may be associated with increased extravascular lung water and pulmonary microvascular permeability, which is the hallmark of lung pathophysiology.

- The categories of the Berlin definition of disease progression are also associated with the severity of physiological derangements and the incidence of organ failure.

\section{Abbreviations}

AECC: American-European Consensus Conference; ALI: acute lung injury; APACHE: acute physiology and chronic health evaluation; APVR: airway pressure release ventilation; ARDS: acute respiratory distress syndrome; BNP: B-type natriuretic peptide; CT: computed tomography; EVLW: extravascular lung water; EVLWi: extravascular lung water index; GEDV: global end-diastolic volume; ITBV: intrathoracic blood volume; ITTV: intrathoracic thermal volume; LIS: lung injury scale; PEEP: positive end-expiratory pressure; P/F ratio: $\mathrm{PaO}_{2}$ / $\mathrm{FiO}_{2}$ ratio; PTV: pulmonary thermal volume; PVPI: pulmonary vascular permeability index; SIRS: systemic inflammatory response syndrome; SOFA: sequential organ failure assessment.

\section{Competing interests}

Dr. Taira is a member of the medical advisory board of Pulsion Medical Systems. The other authors declare no competing interests.

\section{Authors' contributions}

All authors conceived and designed the study, wrote the study protocol, and acquired the clinical data. SK was responsible for the statistical analyses and the first draft of the manuscript. All authors amended and commented on the manuscript and approved the final version. 


\section{Acknowledgements}

This work was supported in part by a Grant-in-Aid for Scientific Research (22592023) from the Ministry of Education, Culture, Sports, Science and Technology of Japan.

This prospective, observational, multi-institutional study was approved by the ethics committees of each of the 23 institutions involved: Tohoku University Graduate School of Medicine, St. Marianna University School of Medicine, Kansai Medical University, Nara Medical University, Kurume University School of Medicine, Fukuoka University, Nippon Medical School Hospital, Nihon University School of Medicine Itabashi Hospital, Tokyo Medical and Dental University Hospital of Medicine, Juntendo University Nerima Hospital, Jikei University School of Medicine, National Hospital Organization Disaster Medical Center, Saiseikai Yokohamashi Tobu Hospital, Social Insurance Chukyo Hospital, Kansai Medical University Takii Hospital, Osaka City General Hospital, Kobe City Medical Center General Hospital, Hiroshima City Hospital, Yamaguchi University Hospital, Nagasaki University Hospital, Nippon Medical School Tama Nagayama Hospital, Nippon Medical School Chiba Hokusou Hospital.

\section{Authors' details}

'Division of Emergency Medicine, Tohoku University Graduate School of Medicine, 2-1, Seiryo-machi, Aoba-ku, Sendai, Miyagi 980-8575, Japan. ${ }^{2}$ Department of Emergency and Critical Care Medicine, Tohoku University Hospital, 1-1 Seiryo-cho, Aoba-ku, Sendai, Miyagi 980-8574, Japan. ${ }^{3}$ Department of Emergency and Critical Care Medicine, Kurume University School of Medicine, 67 Asahi-machi, Kurume-shi, Fukuoka 830-0011, Japan. ${ }^{4}$ Department of Emergency and Critical Care Medicine, Faculty of Medicine, Fukuoka University, 8-19-1 Nanakuma, Jonan-ku, Fukuoka 814-0180, Japan. ${ }^{5}$ Department of Emergency and Critical Care Medicine, Kansai Medical University, 10-15 Fumizonocho, Moriguchi, Osaka 573-1191, Japan. ${ }^{6}$ Department of Emergency and Critical Care Medicine, St. Marianna University School of Medicine, 2-16-1 Sugao, Miyamae-ku, Kawasaki-shi, Kanagawa 216-8511, Japan. ${ }^{7}$ Department of Emergency and Critical Care Medicine, Nara Medical University, 840 Shijo-cho, Kashihara, Nara 634-8521, Japan. ${ }^{8}$ Department of Emergency and Critical Care Medicine, Nippon Medical School Hospital, 1-1-5 Sendagi, Bunkyo-ku, Tokyo 113-8603, Japan. ${ }^{9}$ Department of Emergency and Critical Care Medicine, Nihon University School of Medicine Itabashi Hospital, 30-1, Oyaguchi Kami-cho, Itabashi-ku, Tokyo 173-8610, Japan. ${ }^{10}$ Shock Trauma and Emergency Medical Center, Tokyo Medical and Dental University Hospital of Medicine, 1-5-45 Yushima, Bunkyo-ku, Tokyo 113-8510, Japan. ${ }^{11}$ Department of Emergency and Critical Care Medicine, Juntendo University Nerima Hospital, 3-1-10 Takanodai, Nerima-ku Tokyo 177-8521, Japan. ${ }^{12}$ Critical Care Medicine, Jikei University School of Medicine, 3-25-8 Nishi-shimbashi, Minato-ku, Tochigi 105 8461, Japan. ${ }^{13}$ Emergency and Critical Care Medicine, National Hospital Organization Disaster Medical Center, 2-5-1, Higashigaoka, Meguro-ku, Tokyo 152-8902, Japan. ${ }^{14}$ Department of Intensive Care Medicine, Saiseikai Yokohamashi Tobu Hospital, 3-6-1 Shimosueyoshi, Tsurumi-ku, Yokohama-shi, Kanagawa 230-8765, Japan. ${ }^{15}$ Department of Emergency and Critical Care Medicine, Social Insurance Chukyo Hospital, 1-1-10. Sanjyo, Minami-ku, Nagoya, Aichi 457-8510, Japan. ${ }^{16}$ Advanced Emergency and Critical Care Center, Kansai Medical University Takii Hospital, 10-15, Fumizono-cho, Moriguchi, Osaka 570-8507, Japan. ${ }^{17}$ Emergency and Critical Care Medical Center, Osaka City General Hospital, 1-5-7 Asahimachi, Abeno-ku, Osaka 5458585, Japan. ${ }^{18}$ Intensive Care Unit, Kobe City Medical Center General Hospital, 4-6, Minatojimanakamachi, Chuo-ku, Kobe, Hyogo 650-0046, Japan. ${ }^{19}$ Department of Anesthesia and Intensive Care, Hiroshima City Hospital, 7-33 Motomachi Naka-ku, Hiroshima-shi, Hiroshima 730-8518, Japan. ${ }^{20}$ Advanced Medical Emergency and Critical Care Center, Yamaguchi University Hospital, 1-1-1, Minamikogushi, Ube-ku, Yamaguchi 755-8505, Japan. ${ }^{21}$ Intensive Care Unit, Nagasaki University Hospital, 1-14 Bunkyo-machi, Nagasaki-ku Nagasak 852-8521, Japan. ${ }^{22}$ Department of Emergency and Critical Care Medicine, Nippon Medical School Tama Nagayama Hospital, 1-7-1 Nagayama, Tamashi, Tokyo 206-8512, Japan. ${ }^{23}$ Department of Emergency and Critical Care Medicine, Nippon Medical School Chiba Hokusou Hospital, 1715 Kamagari, Inzai-shi, Chiba 270-1694, Japan.

Received: 31 January 2013 Revised: 6 April 2013 Accepted: 20 June 2013 Published: 20 June 2013

\section{References}

1. Ashbaugh $D G$, Bigelow DB, Petty $T L$, Levine BE: Acute respiratory distress in adults. Lancet 1967, 2:319-323.

2. Bernard GR, Artigas A, Brigham KL, Carlet J, Falke K, Hudson L, Lamy M, Legall JR, Morris A, Spragg R: The American-European Consensus Conference on ARDS: definitions, mechanisms, relevant outcomes, and clinical trial coordination. Am J Respir Crit Care Med 1994, 149:818-824.

3. Ferguson ND, Davis AM, Slutsky AS, Stewart TE: Development of a clinical definition for acute respiratory distress syndrome using the Delphi technique. J Crit Care 2005, 20:147-154.

4. Neff MJ: The epidemiology and definition of the acute respiratory distress syndrome. Respir Care Clin N Am 2003, 9:273-282.

5. Estenssoro E, Dubin A, Laffaire E, Canales HS, Sáenz G, Moseinco M, Bachetti P: Impact of positive end-expiratory pressure on the definition of acute respiratory distress syndrome. Intensive Care Med 2003, 29:1936-1942.

6. Phua J, Stewart TE, Ferguson ND: Acute respiratory distress syndrome 40 years later: time to revisit its definition. Crit Care Med 2008, 36:2912-2921.

7. Rubenfeld GD, Caldwell E, Granton J, Hudson LD, Matthay MA: Interobserver variability in applying a radiographic definition for ARDS. Chest 1999, 116:1347-1353.

8. Meade MO, Cook RJ, Guyatt GH, Groll R, Kachura JR, Bedard M, Cook DJ, Slutsky AS, Stewart TE: Interobserver variation in interpreting chest radiographs for the diagnosis of acute respiratory distress syndrome. Am J Respir Crit Care Med 2000, 161:85-90.

9. Martin GS, Eaton S, Mealer M, Moss M: Extravascular lung water in patients with severe sepsis: A prospective cohort study. Crit Care 2005, 9: R74-R82.

10. Phillips CR, Smith SM: Predicted body weight-indexed extravascular lung water is elevated in acute respiratory distress syndrome. Crit Care Med 2009, 37:377-378.

11. Davey-Quinn A, Gedney JA, Whiteley SM, Bellamy MC: Extravascular lung water and acute respiratory distress syndrome-oxygenation and outcome. Anaesth Intensive Care 1999, 27:357-362.

12. The ARDS Definition Task Force: Acute respiratory distress syndromethe Berlin Definition. JAMA 2012, 307:2526-2533.

13. Halperin BD, Feeley TW, Mihm FG, Chiles C, Guthaner DF, Blank NE: Evaluation of the portable chest roentgenogram for quantitating extravascular lung water in critically ill adults. Chest 1985, 88:649-652.

14. Lindqvist B: Experimental uraemic pulmonary oedema including: criteria for pulmonary oedema in anuric rabbits, the role of uremia and overhydration, and a literary survey on the problems of uraemic pulmonary oedema (fluid-retention lung, etc.). Acta Med Scand 1964, 176:1.

15. Mihm FG, Feeley TW, Rosenthal MH, Lewis F: Measurement of extravascular lung water in dogs using the thermal-green dye indicator dilution method. Anesthesiology 1982, 57:116-122.

16. Mihm FG, Feeley TW, Jamieson SW: Thermal dye double indicator dilution measurement of lung water in man: comparison with gravimetric measurements. Thorax 1987, 42:72-76.

17. Sakka SG, Rühl CC, Pfeiffer UJ, Beale R, McLuckie A, Reinhart K, MeierHellmann A: Assessment of cardiac preload and extravascular lung water by single transpulmonary thermodilution. Intensive Care Med 2000, 26:180-187.

18. Neumann P: Extravascular lung water and intrathoracic blood volume: double versus single indicator dilution technique. Intensive Care Med 1999, 25:216-219.

19. Tagami T, Kushimoto S, Yamamoto Y, Atsumi T, Tosa R, Matsuda K, Oyama R, Kawaguchi T, Masuno T, Hirama H, Yokota H: Validation of extravascular lung water measurement by single transpulmonary thermodilution: human autopsy study. Crit Care 2010, 14:R162.

20. Katzenelson R, Perel A, Berkenstadt H, Preisman S, Kogan S, Sternik L, Segal E: Accuracy of transpulmonary thermodilution versus gravimetric measurement of extravascular lung water. Crit Care Med 2004, 32:1550-1554.

21. Kushimoto S, Taira Y, Kitazawa Y, Okuchi K, Sakamoto T, Ishikura H, Endo T, Yamanouchi S, Tagami T, Yamaguchi J, Yoshikawa K, Sugita M, Kase Y, Kanemura T, Kuroki Y, Izumino H, Rinka H, Seo R, Takatori M, Kaneko T, Nakamura T, Irahara T, Saitou N, Watanabe A, The PiCCO Pulmonary Edema 
Study Group: The clinical usefulness of extravascular lung water and pulmonary vascular permeability index to diagnose and characterize pulmonary edema: a prospective multicenter study on the quantitative differential diagnostic definition for acute lung injury/acute respiratory distress syndrome. Crit Care 2012, 16:R232.

22. Michard F: Bedside assessment of extravascular lung water by dilution methods: temptations and pitfalls. Crit Care Med 2007, 35:1186-1192.

23. LeTourneau JL, Pinney J, Phillips CR: Extravascular lung water predicts progression to acute lung injury in patients with increased risk. Crit Care Med 2012, 40:847-854.

24. Newman EV, Merrell M, Genecin A, Monge C, Milnor WR, McKeever WP: The dye dilution method for describing the central circulation. An analysis of factors shaping the time-concentration curves. Circulation 1951, 4:735-746.

25. Monnet X, Anguel N, Osman D, Hamzaoui O, Richard C, Teboul JL: Assessing pulmonary permeability by transpulmonary thermodilution allows differentiation of hydrostatic pulmonary edema from ALI/ARDS. Intensive Care Med 2007, 33:448-453.

26. Michard F, Schachtrupp A, Toens C: Factors influencing the estimation of extravascular lung water by transpulmonary thermodilution in critically ill patients. Crit Care Med 2005, 33:1243-1247.

27. Tagami T, Kushimoto S, Tosa R, Omura M, Hagiwara J, Hirama H, Yokota H: The precision of $\mathrm{PiCCO}^{\circledast}$ measurements in hypothermic post-cardiac arrest patients. Anaesthesia 2012, 67:236-243.

28. Crapo RO, Morris AH, Gardner RM: Reference spirometric values using techniques and equipment that meet ATS recommendations. Am Rev Respir Dis 1981, 123:659-664.

29. O'Brien JM Jr, Phillips GS, Ali NA, Lucarelli M, Marsh CB, Lemeshow S: Body mass index is independently associated with hospital mortality in mechanically ventilated adults with acute lung injury. Crit Care Med 2006, 34:738-744.

30. Berkowitz D, Danai P, Eaton S, Moss M, Martin GS: Accurate characterization of extravascular lung water in acute respiratory distress syndrome. Crit Care Med 2008, 36:1803-1809.

31. The Acute Respiratory Distress Syndrome Network: Ventilation with lower tidal volumes as compared with traditional tidal volumes for acute lung injury and the acute respiratory distress syndrome. N Engl J Med 2000, 342:1301-1308.

32. Phillips CR, Chesnutt MS, Smith SM: Extravascular lung water in sepsisassociated acute respiratory distress syndrome: indexing with predicted body weight improves correlation with severity of illness and survival. Crit Care Med 2008, 36:69-73.

33. Craig TR, Duffy MJ, Shyamsundar M, McDowell C, McLaughlin B, Elborn JS, McAuley DF: Extravascular lung water indexed to predicted body weight is a novel predictor of intensive care unit mortality in patients with acute lung injury. Crit Care Med 2010, 38:114-120.

34. Knaus WA, Draper EA, Wanger DP, Zimmerman JE: APACHE II: a severity classification system. Crit Care Med 1985, 13:818-829.

35. Ferreira FL, Bota DP, Bross A, Mélot C, Vincent JL: Serial evaluation of the SOFA score to predict outcome in critically ill patients. JAMA 2001, 286:1754-1758.

36. Murray JF, Matthay MA, Luce JM, Flick MR: An expanded definition of the adult respiratory distress syndrome. Am Rev Respir Dis 1998, 138:720-723.

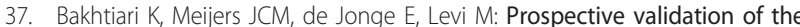
International Society of Thrombosis and Haemostasis scoring system for disseminated intravascular coagulation. Crit Care Med 2004, 32:2416-2421.

38. Craig TR, Duffy MJ, Shyamsundar M, McDowell C, McLaughlin B, Elborn JS, McAuley DF: Extravascular lung water indexed to predicted body weight is a novel predictor of intensive care unit mortality in patients with acute lung injury. Crit Care Med 2010, 38:114-120.

doi:10.1186/cc12811

Cite this article as: Kushimoto et al:: Relationship between extravascular lung water and severity categories of acute respiratory distress syndrome by the Berlin definition. Critical Care 2013 17:R132.

\section{Submit your next manuscript to BioMed Central and take full advantage of:}

- Convenient online submission

- Thorough peer review

- No space constraints or color figure charges

- Immediate publication on acceptance

- Inclusion in PubMed, CAS, Scopus and Google Scholar

- Research which is freely available for redistribution

Submit your manuscript at www.biomedcentral.com/submit
C Biomed Central 